\title{
A rare surgical emergency - trans anal evisceration of small intestine
}

\author{
Subramaniyam Raviraj ${ }^{1}$, Ratnasingam Vaheesan ${ }^{2}$ \\ ${ }^{1}$ Faculty of Medicine, University of Jaffna, Sri Lanka \\ ${ }^{2}$ District hospital Mullaitivu, Sri Lanka
}

Key words: Trans anal; laparotomy; evisceration

\section{Introduction}

A trans anal evisceration of the small intestine through the anus is a rare surgical emergency [1,2]. This surgical condition may occur spontaneously or associate with rectal injuries [1]. According to the literature, blunt abdominal or pelvic trauma is the main cause for the transanal small bowel evisceration in young patients [1].

There were several case reports in the journals indicating the association between the rectal prolapse and trans anal evisceration of the small bowel as in this patient $[2,3]$. This case report, it illustrates aetiology, management and complications of trans anal evisceration of the small intestine.

\section{Presentation}

A 72-year-old woman transferred from District General Hospital Mullaitivu after an emergency laparotomy to teaching Hospital Jaffna for further management.

This patient was admitted to Mullaithevu District General Hospital with the history of the evisceration of the small intestinal loop through the anus for the last 2 hours. She had no history of recent trauma to abdomen. But she admitted that she had prolapsed rectum and reduction done one year ago at Mullaithevu District General Hospital.

Her bowel habits were normal but there is a history of rectal prolapse 1 year before this admission and reduction done at District General Hospital Mullaithevu. There is no history suggestive of inflammatory bowel disease or colorectal malignancy. The emergency laparotomy was performed at D.G.H Mullaithevu after optimized the patient. The emergency laparotomy revealed there was a laceration in the anterior rectal wall and small intestinal herniation through this defect. The adhesions have been noted between small bowel loops and edges of rectal wall defect. Adhesions were separated and accidental damage to the small intestinal loop was noted during the reduction of herniated small bowel loop. The resection and anastomosis of the small intestine were

Correspondence: Subramaniyam Raviraj

E-mail: dr.s.raviraj@gmail.com

Received: 11-06-2019 Accepted: 18-10-2019

(iD http://orcid.org/000-0001-6893-2662

DOI: http://doi.org/10.4038/sljs.v37i3.8595

The Sri Lanka Journal of Surgery 2019; 37(3): 4- 5

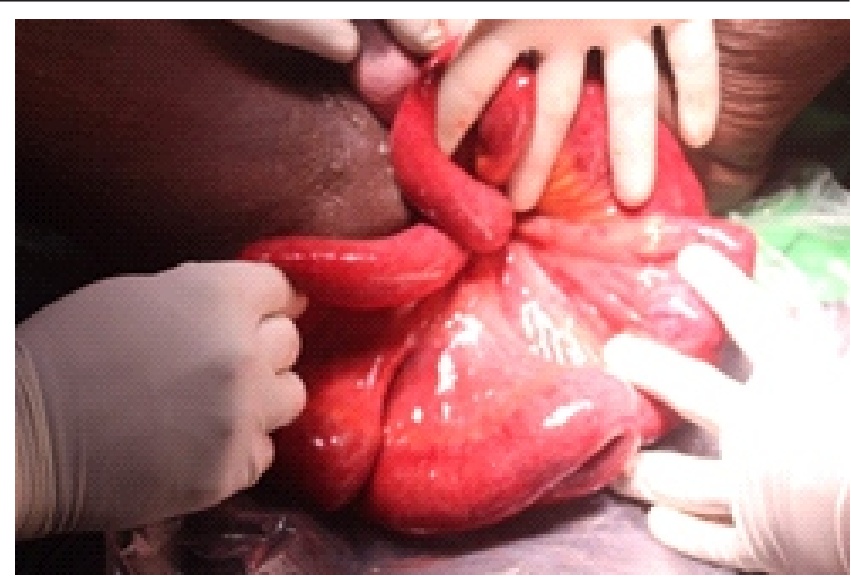

Figure 1. Eviscerated part of small intestine with mesentery.

carried out. The anterior rectal wall defect was repaired and sigmoid colostomy (Double Barrel] was done. Routine closure of the abdomen with intraperitoneal drainage tube was done. Then she was transferred to the Teaching Hospital Jaffna on the 5th post-operative day for the management of sepsis. The CRP and Full Blood Count suggested sepsis. The ultrasound scan of abdomen and pelvis revealed a small amount of fluid collection in the pelvic cavity.

The contrast-enhanced CT of the abdomen and pelvis revealed there was no anastomotic leakage from the anastomotic site or elsewhere but a small amount of fluid collection in the pelvic cavity. The patient died on 10th postop day due to multi-organ failure due to sepsis.

\section{Discussion}

The transanal evisceration of the small bowel is an extremely rare complication $[1,2]$. This may occur either due to Abdominal and Pelvic trauma especially in young patients or as a result of chronic prolapse of rectum $[1,2]$. This surgical emergency occurs as a result of herniation of small bowel through the breach in the rectal wall. Less than 70 similar cases have been reported in various literature [1]. The first recorded case is in 1827 by Brodie. Since then very few cases have been reported up to now.

Majority of the cases reported in the literature occur as a spontaneous event following a chronic rectal prolapse, especially in elderly patients as in our patient [1]. Less than 20 cases have been documented as a result of rectal traumatic 


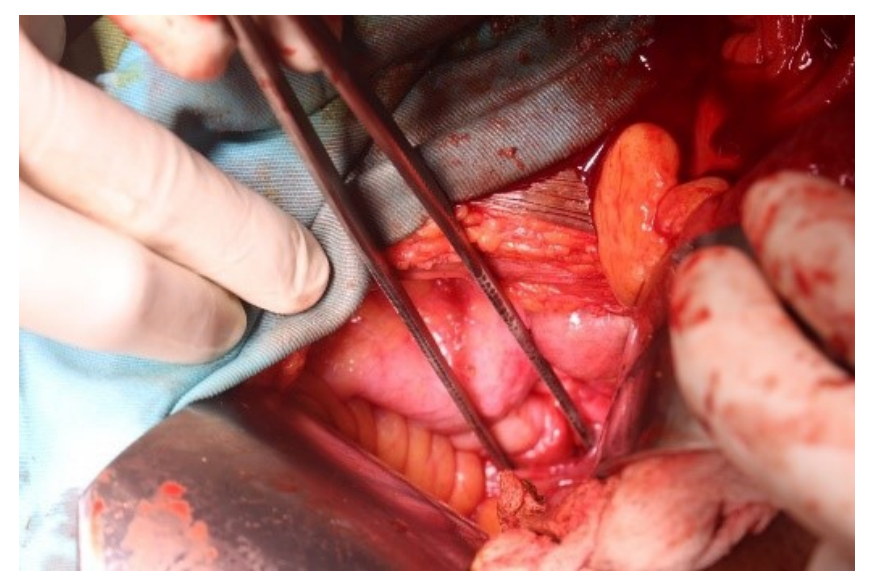

Figure 2. Evisceration occurred through Anal orifice.

injuries in young patients [1]. Wroblesk DE, Duley TH reported that mainly two factors predispose for this unusual clinical scenario [4]. More than $75 \%$ of these cases were due to the chronic prolapse of rectum and an event of sudden increase intraabdominal pressure result in a spontaneous rupture which commonly occurs in the anterior rectal wall as in this patient [3].

Broden B, Snellman B with cine radiographic studies explained that the basis of prolapse rectum is due to the Sliding Hernia of small bowel into the pouch of Douglas which forms a hernial sac with its contents invaginate the anterior wall of the rectum. Invaginating of the rectal anterior wall by hernial sac with its content lead to ischemia of the rectal anterior wall and makes the anterior wall the weakest point. Finally, the anterior wall of the rectum becomes more vulnerable for perforation allowing the small bowel to herniate and eviscerate through the anal canal. The other causes for the transanal evisceration of bowel without preexisting chronic rectal prolapse have been reported secondary due to blunt abdominal wall injury or iatrogenic injury or due to suction injuries mainly in children [4].

The management of both types of trans anal evisceration of bowel depends on viability, perforation and contamination of herniated bowel [1]. Eviscerated bowel should be cleaned with warm saline and gentle reduction into the peritoneal cavity attempted under the general anaesthesia but however is rarely possible..
This can cause higher mortality. Attempts to reduce the bowel through the anal canal without a laparotomy is unsuccessful most of the time and can cause perforation of eviscerated bowel as in this patient [4].

Laparotomy with careful reduction of eviscerated bowel reduces the mortality. The gangrenous or perforated bowel may need resection and anastomosis similar to this patient. A rectal tear may need primary repair with proximal loop colostomy. The primary treatment such as Rectopexy or sigmoid colectomy to be considered. Hysterectomy should be considered when the trans anal evisceration of the small bowel is associated with uterine prolapse to prevent further recurrence [5].

\section{Conclusion}

The transanal evisceration of the small intestine is a rare presentation. It may occur as a result of chronic rectal prolapse or rectal injuries. A clear understanding of the pathophysiology of this condition would help in efficient and prompt response in an emergency.

All authors disclose no conflict of interest. The study was conducted in accordance with the ethical standards of the relevant institutional or national ethics committee and the Helsinki Declaration of 1975, as revised in 2000 .

\section{References}

1. Trans anal evisceration of the small bowel from blunt abdominal trauma. A.C Adisa, C Onyegbule and A.U Mbanaso. Nigerian Journal of Surgical Research, Vol 8 No. 3-4, 2006: 182 - 184.

2. Transanal Evisceration of Small Bowel - A Rare Surgical Emergency. Narayana Swamy Chetty Y.V, Sridhar M and Pankaja S.S. https://doi.org/10.7860/JCDR/2014/7231.3969

3. Wrobleski DE, Dailey TH. Spontaneous rupture of the distal colon with evisceration of small intestine through the anus; report of two cases and review of literature. Dis Colon Rectum. 1979; 22:569-57. https://doi.org/10.1007/BF02587009

4. Neil R, Price, et Swimming pool filter-induced trans rectal evisceration in children: Australian experience. Med J Aust. 2010; 192(9):534-36.

https://doi.org/10.5694/j.1326-5377.2010.tb03621.x

5. Joon Joeng, et al. Rupture of recto sigmoid colon with evisceration of the small bowel through anus. Yonesi Medical Journal. 2000; 41(2):289-92. https://doi.org/10.3349/ymj.2000.41.2.289

\section{Learning Points:}

- The basic pathology of this emergency rare surgical condition is an important factor in the management.

- Even though it's a rare condition the principle of management is necessary to overcome this emergency. 